\title{
Wnt and GSK3 Signaling Pathways in Bipolar Disorder: Clinical and Therapeutic Implications
}

\author{
Ather Muneer \\ Department of Psychiatry, Islamic International Medical College, Riphah International University, Rawalpindi, Pakistan
}

\begin{abstract}
The neurobiology of bipolar disorder, a chronic and systemic ailment is not completely understood. The bipolar phenotype manifests in myriad ways, and psychopharmacological agents like lithium have long term beneficial effects. The enzyme glycogen synthase kinase 3 (GSK3) has come into focus, as lithium and several other mood stabilizing medications inhibit its activity. This kinase and its key upstream modulator, Wnt are dysregulated in mood disorders and there is a growing impetus to delineate the chief substrates involved in the development of these illnesses. In May 2016, a comprehensive literature search was undertaken which revealed that there is over activity of GSK3 in bipolar disorder with deleterious downstream effects like proinflammatory status, increased oxidative stress, and circadian dysregulation leading to declining neurotrophic support and enhanced apoptosis of neural elements. By developing specific GSK3 inhibitors the progressive worsening in bipolar disorder can be forestalled with improved prospects for the sufferers.
\end{abstract}

KEY WORDS: Bipolar disorder; Glycogen synthase kinase 3; Wnt; Protein kinase B; $\beta$-catenin; Canonical Wnt pathway.

\section{INTRODUCTION}

Bipolar disorder (BD) is a chronic disease with hitherto unknown etiology and pathophysiology. It can be conceptualized as classical BD in which the sufferers have a positive family history, discrete manic and depressive episodes, good inter-episode functioning and responsiveness to the standard mood stabilizer, lithium. In contrast, patients with the bipolar spectrum illness are afflicted with mixed episodes, rapid cycling and persistent subthreshold affective symptoms. They have history of psychotic spectrum disorders in first degree relatives, high rates of comorbid anxiety and substance use disorders and poor treatment response to conventional mood stabilizers. Whereas, the illness trajectory of classical BD patients is benign, the disorder takes a neuroprogressive course in bipolar spectrum cases with cognitive and functional impairments in the latter stages of the ailment. BD patients with the classical illness constitute only $30 \%$ of the cases, while the majority have the more severe and refractory

Received: August 29, 2016 / Accepted: November 20, 2016 Address for correspondence: Ather Muneer, MBBS, FCPS Department of Psychiatry, Islamic International Medical College, Riphah International University, 274 Peshawar Road, Rawalpindi, Pakistan

Tel: +92-51-548-1828, Fax: +92-51-512-5170

E-mail: muneerather2@gmail.com diathesis. The lithium insensitive bipolar subjects undergo polypharmacy, treatment emergent adverse effects and compliance problems, while their prognosis is negatively affected by such illness factors as increased severity and frequency of episodes. ${ }^{1)}$

The current understanding of the neurobiology of BD expounds that the illness phenotype is expressed in genetically predisposed individuals, with biologically and psychologically mediated stress acting as the precipitating factor. Abnormalities in homeostatic balance have been substantiated as the pathophysiological underpinnings which cause the initiation and progression of the disorder. Affective exacerbations are marked by loss of diurnal variation of cortisol, increased circulating pro-inflammatory cytokines, oxidative and nitrosative stress. In the brain, there is evidence of glutamate exicitotoxicity, loss of inhibitory phenotype of gabaergic interneurons and increased apoptosis in key mood regulating areas like the hippocampus. An understanding of the molecular events that underlie these deleterious changes is essential for better therapeutics of an otherwise intractable condition. Research endeavors have identified several promising targets, for instance glucorticoid receptor chaperone proteins, enzymes in the kynurenine metabolic pathway and NADPH oxidases. ${ }^{2)}$ Knowledge regarding the mode of action of lithium, and other psychotropic drugs used in the

(c) This is an Open-Access article distributed under the terms of the Creative Commons Attribution Non-Commercial License (http://creativecommons.org/licenses/by-nc/4.0) which permits unrestricted non-commercial use, distribution, and reproduction in any medium, provided the original work is properly cited. 
treatment of affective disorders has further elucidated the neurobiological mechanisms underlying these conditions.

Consistent research efforts have validated the pivotal role for glycogen synthase kinase 3 (GSK3) $\beta$ in the pathogenesis of mood disorders in general, and BD in particular. The initial reports that GSK3 is implicated in affective illnesses date back to 1996 when two separate groups (Klein and Melton; Stambolic et al.) described the direct inhibitory effect of lithium on GSK3 by a magnesium-competitive mechanism. The key role that this enzyme plays in BD pathogenesis is supported by the following observations, as described elaborately in the article by $\mathrm{Cole}^{3)}$ :

1) Lithium, the prototype mood stabilizer is both a direct and indirect inhibitor of GSK3.

2) In rat models of mania, specific GSK 3 inhibitors reproduce behaviors mimicking effects of lithium.

3) Genetic modification of GSK3 activity in mice results in behaviors that are reminiscent of BD.

4) Mice with decreased GSK3 activity $\left(G S K 3 \beta^{+/-}\right.$; $G S K 3 \alpha^{-1-}$ ) exhibit behaviors that replicate chronic lithium treatment.

5) Mice that overexpress GSK3 $\beta$ show hyperactivity, correlating with mania in humans.

6) Overexpression of GSK3 $\beta$ annuls the ameliorative behavioral effects caused by lithium treatment in mice.

7) In humans, polymorphisms in the GSK $3 \beta$ promoter region are linked to earlier age at onset of $\mathrm{BD}$, therapeutic sensitivity to lithium and white matter microstructure anomalies (white matter hyperintensities).

8) Finally, upstream regulators of GSK $3 \beta$ like Dorsophila wingless proteins (Wnt), disrupted in schizophrenia 1 and protein kinase B (Akt) are genetically associated with mood disorders.

Affective episodes, in particular manic exacerbations are associated with overexpression of pro-apoptotic factors (Bax, BAD, caspase-9 and caspase-3) and decline of anti-apoptotic proteins like brain-derived neurotrophic factor (BDNF) and Bcl-2. ${ }^{4}$ Wnt molecules regulate crucial processes in the development of mammalian nervous system, for instance patterning, cell fate specification, proliferation and neuronal morphology. In adults, Wnt ligands play a vital role in the propagation and differentiation of neural progenitors to form fresh cells which can then migrate, mature and integrate into brain circuits. Wnt signaling involves the inhibition of the constitutively active intracellular enzyme GSK3 $\beta$, leading to the stabilization and nuclear translocation of the transcriptional co-activator, $\beta$-catenin. In the nucleus, $\beta$-catenin interacts with the lymphocyte enhancer factor/T-cell factor (LEF/TCF) family of transcription factors to activate expression of genes involved in neurotrophic support, oxidative stress regulation, circadian cycle and maintenance of inflammatory homeostasis. This is referred to as the canonical pathway and plays an overarching role in the functioning of the mammalian nervous system. ${ }^{5}$

With this preamble, the rationale of the review is to draw attention to the most recent developments in this area. To accomplish the stated objective, the Wnt and GSK3 signaling pathways are characterized in detail followed by their relevance to the pathogenesis of BD. In the final part, the mechanism of action of psychotherapeutic drugs used in the treatment of BD is outlined and future directions are delineated.

\section{MAIN SUBJECTS}

\section{Search Strategy}

In May 2016, the PubMed electronic database was searched with several combinations of terms to acquire a comprehensive overview of GSK3/Wnt involvement in BD. Some examples included 'GSK3 and bipolar disorder', 'Wnt and bipolar disorder', 'bipolar disorder and neurogenesis', 'bipolar disorder and apoptosis' and 'lithium and GSK3'. A very large number of articles approximating 800 were retrieved. The search was narrowed to papers published in the last 10 years and this resulted in about 400 citations. The clinical significance of these articles was judged by reading the abstracts, resulting in the exclusion of further 200 papers. About 80 papers were found to be specifically pertinent to the current topic, and included animal and human studies and review articles. These were read in detail and their reference lists were also consulted to gain a composite view of the subject. The information obtained by this strategy was delineated in a concise and coherent style to illuminate the matter under discussion.

\section{GSK3 a Multi-tasking Kinase}

First identified in 1980 and named for its ability to phosphorylate and inactivate the metabolic enzyme glycogen synthase, GSK 3 has since been characterized as a crucial catalyst that modulates several essential neural functions. Two isoforms, GSK $3 \alpha$ and GSK $3 \beta$ are encoded by the human genome which share $85 \%$ sequence homology and although their functions are broadly similar, these are by no means identical. GSK3 $\beta$ is a plieotropic enzyme, expressed throughout the brain with nu- 
merous recognized substrates sub serving roles in such diverse cellular processes as metabolism, neurogenesis, synaptic plasticity and apoptosis. ${ }^{6}$ Intriguingly, for three principal reasons, it is one of most remarkable kinases programmed by the human DNA. Firstly, most if not all substrates need priming by another kinase before these can be targeted by GSK3. Secondly, in contrast to most other kinases it is inherently active in cells under basal conditions and lastly, phosphorylation at an N-terminal serine residue causes inhibition of its kinase activity. As opposed to serine phosphorylation, GSK3 activity is promoted by tyrosine phosphorylation at tyrosine- 279 of GSK $3 \alpha$ and tyrosine- 216 of GSK $3 \beta$, which makes possible contact for substrate binding. This autoposhorylation event of the tyrosine residue on the activation loop of the kinase domain is essential and is most probably intramolecularly mediated and not under regulatory control of upstream modulators. ${ }^{7)}$

\section{Wnt Modulation of GSK3 Activity}

GSK3 regulates multiple signaling pathways such as sonic hedgehog, notch, insulin, neurotrophins (BDNF) and growth factors through protein kinase B or Akt. These mediators activate phosphatidylinositol-3-kinase (PI3K) and Akt which phosphorylates GSK3 at an amino terminal serine residue (Ser21 on GSK $3 \alpha$ and Ser9 on GSK3 $\beta$ ) creating a pseudo-substrate motif that inhibits the enzyme's activity and allows activation of downstream effectors like glycogen synthase and the mammalian target of rapamycin (mTOR). Additionally, GSK3 plays a key inhibitory role in the Wnt pathway. The latter are a family of secreted, cysteine-rich, glycosylated protein ligands that impact on cell growth, differentiation, migration and fate. $^{8)}$

In the canonical pathway, Wnt binds to the transmembrane receptor frizzled $(\mathrm{Fz})$ and its co-partners, low density lipoprotein receptor-related proteins 5 and 6 (LRP5/6), leading to the recruitment of the scaffolding protein, dishevelled (Dvl) and phosphorylation of LRP5/6. In the unstimulated cell, $\beta$-catenin is held in a destruction complex consisting of axin, adenomatous polyposis coli, casein kinase 1 (CK1) and GSK3. Primed by CK1, GSK3 phosphorylates $\beta$-catenin leading to its ubiquitination and proteosomal degradation. In the cell activated by Wnt-Fz-Dvl complex, phosphorylated LRP5/6 disrupts the Axin scaffold preventing GSK3 phosphorylation of $\beta$-catenin and resulting in its accumulation in the cytoplasm. The accrued $\beta$-catenin translocates to the nucleus where it acts together with LEF/TCF family of transcription factors leading to the expression of Wnt target genes. Elucidated by almost two decades of research, the canonical Wnt signaling pathway is being increasingly incriminated in the pathophysiological mechanisms underlying major psychiatric disorders, including BD. ${ }^{9)}$

Figure 1 schematically depicts the inhibitory regulation of GSK3, and Figure 2 is an illustrative representation of the Wnt/ $\beta$-catenin intracellular pathway.

\section{Wnt/Gsk3 Signaling in Mood Disorders; Insights from Animal Models}

Studies in rodents have been very helpful in clarifying the role of $\mathrm{Wnt} / \mathrm{GSK} 3$ pathway in mammalian behavior. In mice, deletion of both copies of GSK3 $\beta$ gene is nonviable and causes death of the animals during gestation; however, haplo-insufficient mice with genotype $G S K 3^{+/-}$ have been engineered. When tested in such validated paradigms as Porsolt's forced swim test, open field and elevated zero maze tests, these experimental animals show behaviors that are reminiscent of mice chronically treated with lithium, a known GSK3 $\beta$ inhibitor. They have decreased immobility time in the forced swim test and reduced exploratory behavior as demonstrated by reduced hole pokes (open field test) and spending of more time in the open segments (elevated zero maze test). Further, in

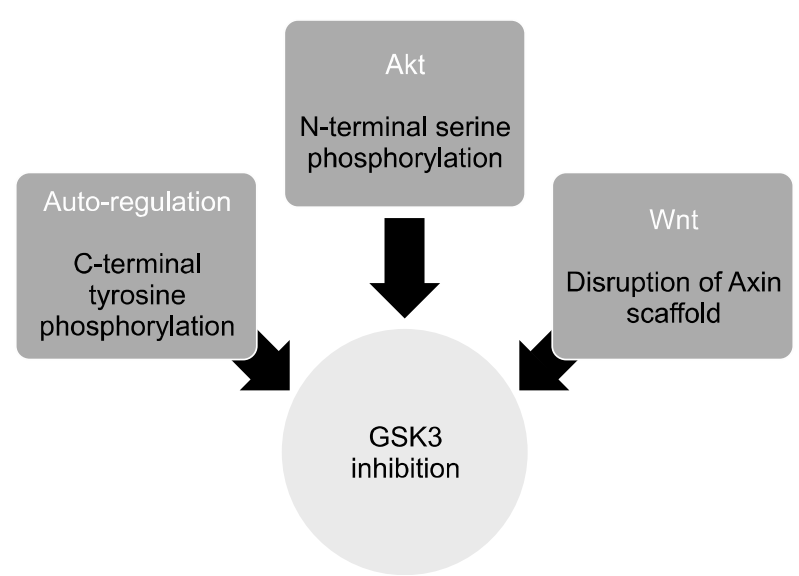

Fig. 1. Inhibitory regulation of glycogen synthase kinase 3 (GSK3). GSK3 is a multi-functioning enzyme that acts as a controlling switch for several crucial intracellular signaling cascades. Insulin/growth factors/neurotrophins, acting through protein kinase B (Akt) cause serine phosphorylation of an $\mathrm{N}$-terminal residue leading to the formation of pseudo-substrate and loss of kinase function of GSK3. Dorsophila wingless protein (Wnt) signaling, on the contrary disrupts the Axin complex in which the constitutively active GSK3 is held in a scaffold. The mood stabilizer, lithium inhibits both of these GSK3 pools; C-terminal tyrosine phosphorylation is most probably an intramolecular event that is considered essential for GSK3 regulation. 
serotonin deficient knockin mice, genetic or pharmacological manipulation of GSK3$\beta$ with resultant inhibition decreases depressive type behaviors. Transgenic mice overexpressing GSK3 $\beta$ by substituting alanine for serine at position 9 show increased locomotor activity, modeling

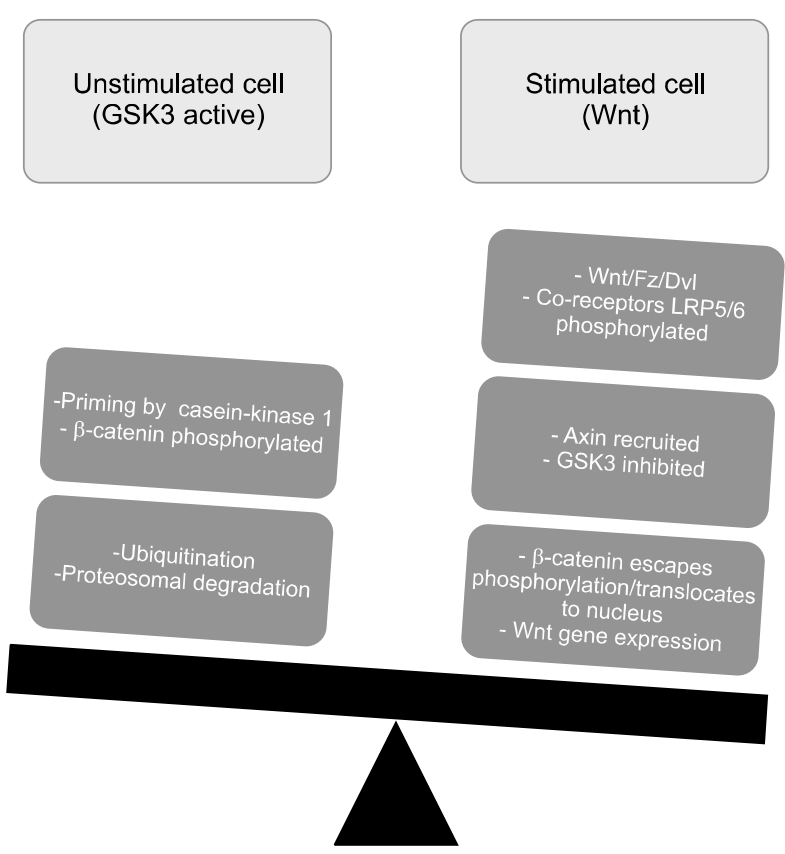

Fig. 2. The canonical Dorsophila wingless protein (Wnt) pathway. Wnt ligands play an essential role in neuronal proliferation, migration and fate. Wnt proteins bind to their transmembrane receptor, frizzled (Fz), recruiting the phospho-protein dishevelled (Dvl) and activating the co-receptors LRP5/6. The later cause disruption of a complex in which the intrinsically active glycogen synthase kinase 3 (GSK3) phosphorylates the transcriptional co-activator, $\beta$-catenin. The unphosphorylated $\beta$-catenin buildsups in the cytoplasm, which facilitates its entry into the nucleus. In the nucleus, $\beta$-catenin interacts with lymphocyte enhancer factor/T-cell factor family of transcription factors, causing expression of the Wnt responsive genes. mania like behavior. Genetically altered mice with an Akt resistant form of GSK $3 \beta$, display decreased anxiety and depressive type behaviors and hyperactivity and enhanced curiosity in various experimental conditions, affording a replicable model of mania. On the contrary, blockade of Dvl function and over-expression of GSK3 $\beta$ in the nucleus accumbens makes mice more vulnerable to social defeat stress and induces behavior mimicking the depression phenotype. Correspondingly, GSK3 $\alpha / \beta$ knockin mice with $\mathrm{N}$-terminal serine to alanine mutations to unblock the kinase activity, show increased liability for behaviors evocative of mood disturbances like amphetamine induced hyperlocomotion and stress provoked depressive behaviors. Finally, as anticipated from the data of transgenic mice presented above, pharmacologic inhibition of GSK3 by psychotropic drugs and small molecule inhibitors decreases both the immobility time in the forced swim test, and amphetamine induced hyperactivity. Taken together, these data strongly implicate Wnt and GSK3 in directing mammalian behavior and point to their pivotal role in mood disorders. The information presented above is well outlined in the review by Jope. ${ }^{10)}$

Table 1 further explains the facts presented in this section and shows that deletions in GSK3 genes, structurally diverse GSK3 inhibitors, lithium and a variety of antidepressants have broadly comparable effect on abnormal behavior phenotypes in mice.

\section{Wnt/GSK3 Pathway Studies in Bipolar Disorder and Related Conditions}

Mood disorders are complex ailments and study of animal behaviors in simple experimental paradigms cannot adequately replicate human diseases. As such there is a growing impetus to investigate the involvement of GSK3 and related pathways in tissues and cells derived from subjects afflicted by BD and associated conditions. The pres-

Table 1. Genetic and pharmacologic manipulation of GSK3 and effects on rodent behavior

\begin{tabular}{|c|c|c|c|c|}
\hline Phenotype & Genetic manipulation & Lithium & Antidepressants & Small molecule GSK3 inhibitors \\
\hline FST (immobility) & $\downarrow G S K 3 \beta^{+/-}$or $G S K 3 \alpha^{-} /^{-}$ & $\downarrow$ & $\downarrow$ & $\begin{array}{l}\downarrow \text { (CHIR99021，AR-A014418, } \\
\text { L803mts) }\end{array}$ \\
\hline Exploratory behavior (hole pokes) & $\downarrow G S K 3^{+1-}$ & $\downarrow$ & No data & No data \\
\hline Elevated zero maze (time in open area) & $\uparrow G S K 3^{+1-}$ & $\uparrow$ & $\uparrow$ & No data \\
\hline TST (immobility) & $\downarrow G S K 3^{-} /^{-}$ & $\downarrow$ & $\downarrow$ & No data \\
\hline Novelty suppressed feeding & No data & $\downarrow$ & $\downarrow$ & No data \\
\hline Novelty induced locomotor activity & $\downarrow G S K 3^{-} /^{-}$ & $\downarrow$ & No data & $\downarrow(T D Z D-8)$ \\
\hline Amphetamine induced hyperlocomotion & $\downarrow \in S K 3 \beta^{+/-}$ & $\downarrow$ & No data & $\begin{array}{l}\downarrow \text { (CHIR99021, TDZD-8, 6-BIO, } \\
\text { AR-A014418, SB216763, } \\
\text { alsterpaullone) }\end{array}$ \\
\hline
\end{tabular}


ent section of the review is dedicated to discussing the findings from the extant literature in this regard.

\section{Post-mortem Studies}

Post-mortem brain studies in bipolar patients indicate that deficits in neurodevelopment and neuroplasticity lie behind the disorder. This is substantiated by the facts that in the sub-genual prefrontal cortex of bipolar subjects, the grey matter is decreased by approximately $40 \%$ and the reduction is due to loss of glial elements and diminution of neuronal numbers and size. Such findings indicate that Wnt signaling pathway which is involved in neurogenesis, patterning and fate, is incriminated in the disease process. ${ }^{11)}$ In a study which measured the protein levels and kinase activities of GSK $3 \beta$ and Akt in the ventral prefrontal cortex area of the brain in 20 suicide victims and 20 drug-free non-suicide subjects, the phosphorylating activity of GSK3 $\beta$ was increased and that of Akt decreased in depressed suicide cases but not in non-depressed victims. ${ }^{12)}$ A more recent study compared protein and mRNA levels of GSK $3 \beta$, pGSK $3 \beta$-ser-9 and $\beta$-catenin in specific brain regions of patients with $\mathrm{BD}$, schizophrenia and normal controls. It showed that both protein and mRNA levels of GSK $3 \beta$ and $\beta$-catenin were decreased in dorsolateral prefrontal cortex and temporal cortex but not the cingulate gyrus in BD subjects, whereas no changes were observed in brains of sufferers with schizophrenia as compared to control cases. This post-mortem investigation implicated Wnt/GSk3 pathway abnormalities in areas of brain with known involvement in mood regulation. ${ }^{13)}$ In another study of noteworthy value, protein and mRNA levels of pGSK $3 \beta$ and $\beta$-catenin were determined in prefrontal cortex and hippocampus of mood disordered teenage suicide victims. Compared to normal controls, the victims had decreased expression of the inhibitory form of GSK $3 \beta$ and $\beta$-catenin in these brain regions, indicating an irregularity in Wnt signaling in teenage suicide. ${ }^{14}$

\section{Studies in Peripheral Blood Cells}

Post-mortem investigation cannot test mood-state dependent alterations in GSK3 activity during manic or depressive episodes, and psychotropic medication use further complicates the issue, making interpretation of findings difficult. An option is to examine blood elements in live patients, for example the peripheral blood mononuclear cells (PBMC). In an attention-grabbing study, PBMC from a small cohort of subjects showed that inhibitory serine phosphorylation of GSK $3 \alpha$ and GSK $3 \beta$ was lower in symptomatic bipolar cases as compared to healthy controls. Intriguingly, this reduction significantly correlated with the severity of manic or depressive exacerbations, indicating that GSK3 activity was influenced by affective states. ${ }^{15)}$ In a study, PBMC from hospitalized acutely manic patients with BD type I were used to assess the protein levels and kinase activity of GSK 3 before and after treatment with mood stabilizers and atypical antipsychotics. In this protocol, the levels of both GSK $3 \alpha$ and GSK $3 \beta$ were increased prior to treatment, while inhibitory serine phosphorylation of the enzyme showed a rise by treatment with lithium, valproate and second generation antipsychotics (SGAs). ${ }^{16)}$ In a more recently published study similar results were obtained in bipolar subjects experiencing major depressive episodes, in whom serine-9 phosphorylated GSK3 $\beta$ ratios increased after lithium therapy and this correlated with symptomatic improvement in the cohort. ${ }^{17)}$ These investigations confirm the notion that GSK3 is involved in the pathophysiology of affective disorders; additionally, mood stabilizing drugs block the activity of GSK3, with this path being involved in the mechanism of action of psychotropic medications.

\section{Genetic Studies}

Genetic studies of the GSK3 enzyme have shed further light on the involvement of this catalytic pathway in phenotypic expression and treatment response in mood disorders. In a case-control study, alterations in the GSK3 $\beta$ gene were studied for single nucleotide polymorphisms (SNP) and copy number variants. Cases consisted of both BD and major depressive disorder (MDD) patients, whereas controls did not have lifetime psychiatric diagnosis of a mood disorder. It was revealed that an SNP and a haplotype in intron 1 of GSK $3 \beta$ gene were associated with earlier age at onset of MDD, implying that these genetic variations may serve as potential markers of severity of mood disorders. ${ }^{18)}$ Monozygotic twins discordant for BD were studied using whole-genome microarrays and reverse transcriptase-polymerase chain reaction. Pathway analysis revealed upregulation of genes involved in Wnt signaling and the biological process of apoptosis in cases as compared to controls. ${ }^{19)}$ A genome-wide association study was conducted in BD subjects with and without history of attempted suicide. It was shown that SNPs on $2 \mathrm{p} 25$ falling in a large linkage disequilibrium block containing the acid phosphatase $1(A C P l)$ gene produced a significant signal in suicide attempters and the expression of this gene was elevated in BD subjects with completed suicide. It is worth mentioning that ACP1 is a protein in- 
criminated in Wnt signaling and lithium, a drug with known anti-suicidal properties is a regulator of this pathway. ${ }^{20)}$ A common comorbidity of BD is increased body mass index (BMI) which is linked to such metabolic disorders as type 2 diabetes mellitus (DM). A genome-wide analysis compared $\mathrm{BD}$ subjects and normal controls with raised BMI as a co-factor, and more than seven hundred thousand SNPs were investigated. In this study, an SNP marker in an intron of transcription factor 7-like2 (TCF7L2) was significantly associated with genetic risk for both $\mathrm{BD}$ and high BMI. The gene, TCF7L2 codes for the transcription factor TCF/LEF, a component of the canonical Wnt pathway and is also one of the strongest genetic risk variants for type $2 \mathrm{DM}$. This study implies that Wnt pathway is involved in the pathogenesis of $\mathrm{BD}$, and the genetic vulnerability in this regard is a shared underlying variation with comorbid metabolic abnormalities of the mood disorder. ${ }^{21)}$

\section{Stem Cell Research}

The latest investigational tool in BD research is stem cells, as it is now possible to reprogram adult somatic tissues to a pluripotent state from which neurons and glia can be derived - the induced pluripotent stem cell (iPSC) models. iPSC lines from bipolar patients and healthy controls have been developed in many laboratory and early interrogations have revealed abnormalities in neuronal differentiation, migration and fate with specific impairments in bipolar patients' originated neurons to dorsal telencephalic derivatives. This points to abnormalities in Wnt, Hedgehog or Nodal signaling pathways which are actively involved in the patterning of central nervous system during development. ${ }^{22)}$ To extend these findings further, a recent study which utilized iPSC lineages from a single family with unaffected parents but two bipolar offspring, revealed multiple phenotypic differences in neural progenitor cell (NPC) development at the level of neurogenesis and expression of genes crucial for neuroplasticity, including Wnt pathway components. ${ }^{23)}$ In summary, recent advances in stem cell biology have made it possible to investigate neurodevelopmental pathways in viable in vitro models from live patients suffering from BD.

The facts presented in this section are further explained in Table 2.

\section{GSK3 Substrates Responsible for Mood Regulation in Bipolar Disorder}

GSK3 is a pleiotropic kinase with many different sub- strates involved in diverse cellular functions. Thus far, more than 90 substrates have been recognized, and confirmed downstream targets include a variety of cytoskeletal, signaling and DNA-binding proteins. While, the prototypic mood stabilizer lithium is a validated GSK3 inhibitor, this action is relatively week, particularly at the therapeutic concentration of around $1 \mathrm{~m}_{\mathrm{M}}$. At this level, perhaps due to differing sensitivity to phosphatases, not all GSK3 substrates are modulated by lithium. Hence, it would be useful to differentiate lithium-sensitive substrates of GSK3 from those that are not influenced by this drug, since the former are liable to be incriminated in the pathogenesis of major psychiatric disorders. Their characterization would be of value in defining the molecular mechanisms underpinning the development of principal mental disorders, and these could themselves become novel therapeutic targets downstream of GSK3 that in theory would be more precise than singling out the kinase itself. $^{24)}$ With this preface, the next sections delineate the most likely substrates linked to the development of mood disorders in general, and BD in particular.

\section{Brain-derived Neurotrophic Factor}

The role of BDNF in mood disorders has received great deal of attention, since this neurotrophic factor is associated with neuronal maturation, differentiation, dendritic growth and synaptic plasticity. With the neuroprogression of illness in $\mathrm{BD}$, there is a decline in BDNF signaling and this may be responsible for loss of grey matter volume in advanced cases of BD. Transcription of BDNF is enhanced by lithium, other GSK3 inhibitors and gene silencing of the kinase, denoting that BDNF expression entails GSK3 inhibition. On the other hand, GSK3 is itself a downstream target of BDNF and binding of this ligand to its receptor tropomyosin receptor kinase $\mathrm{B}$, activates the PI3K-Akt signaling cascade and causes phosphorylation of N-terminal serine residues on GSK $3 \alpha$ and GSK3 $\beta$. Consistent with reduction in BDNF production in severe and repeated mood episodes in BD, the activity of GSK3 and its upstream regulator Akt are increased and decreased, respectively. As such, elevated GSK3 activity is a reason as well as a consequence of diminished BDNF signaling, a main cause of development and advancement of mood disorders. ${ }^{25)}$

\section{Regulation of Neurogenesis}

In adult mammals new neurons arise from NPC in the subgranular zone (SGZ) of the dentate gyrus within the hippocampus and subventricular zone (SVZ) adjacent to 


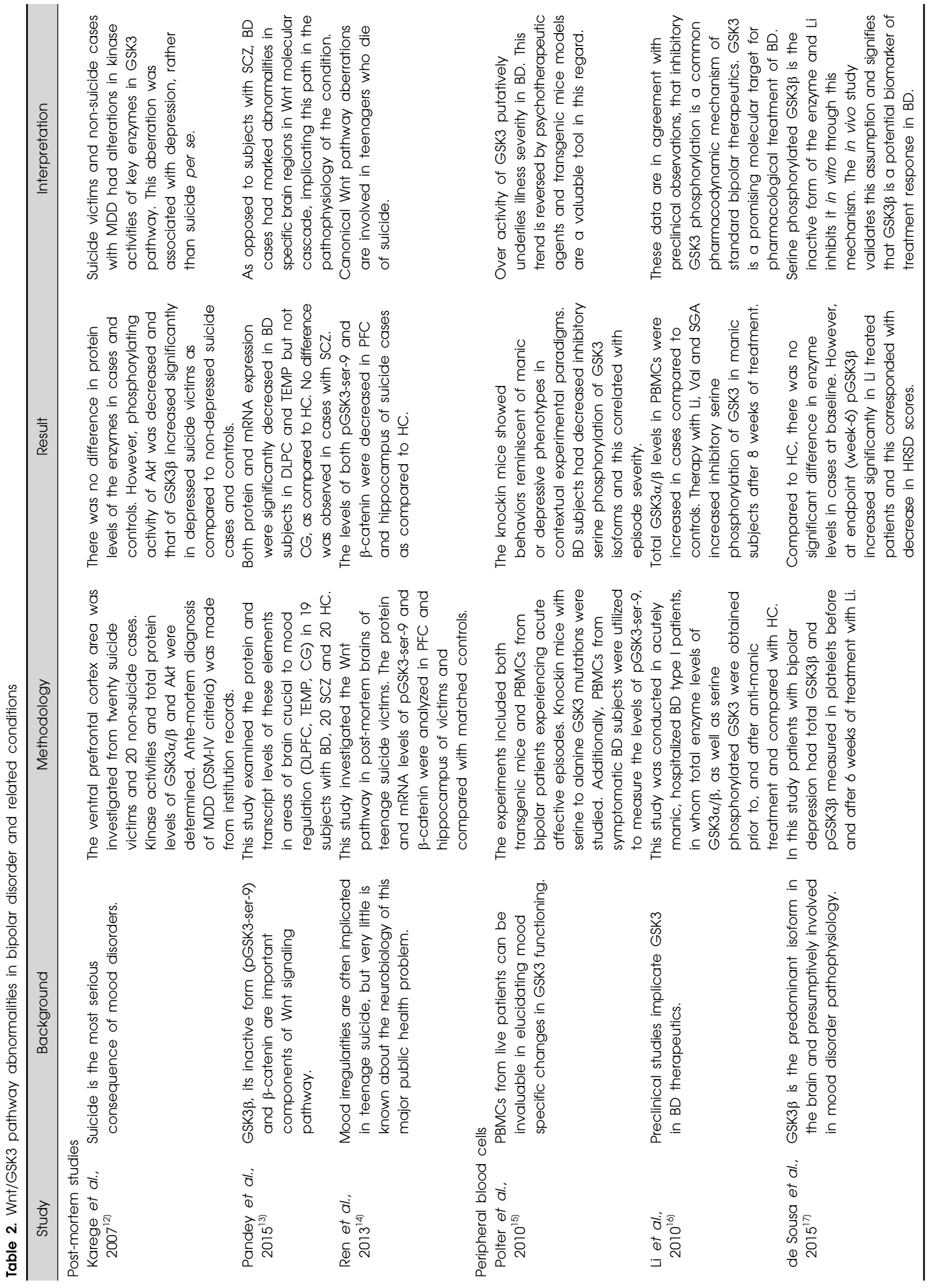




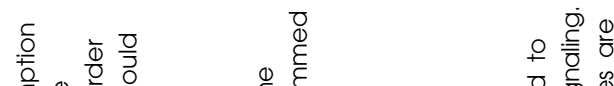

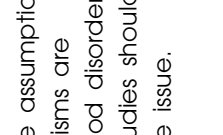

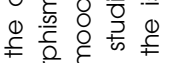

$\stackrel{0}{\ddagger} \sum_{0}^{\circ}$

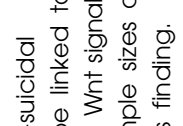

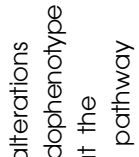

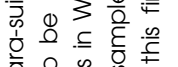

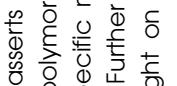

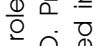

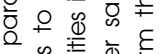

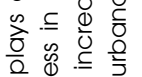

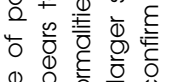

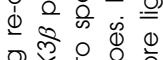

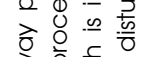

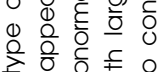

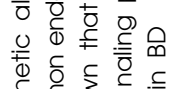

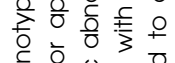

舟

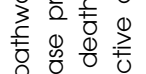

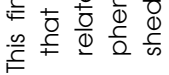

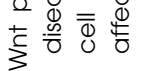

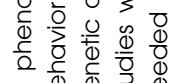

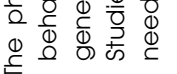

ब.

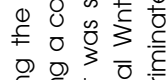

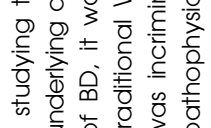

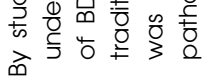

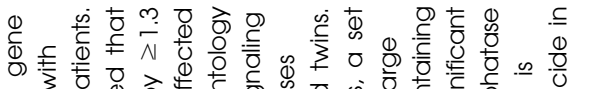

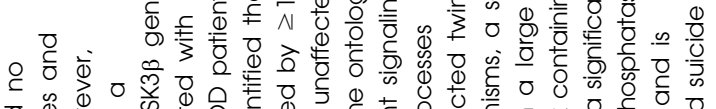

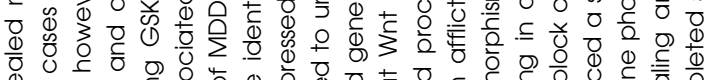

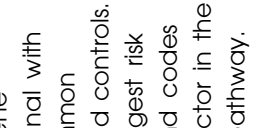

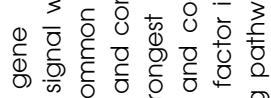

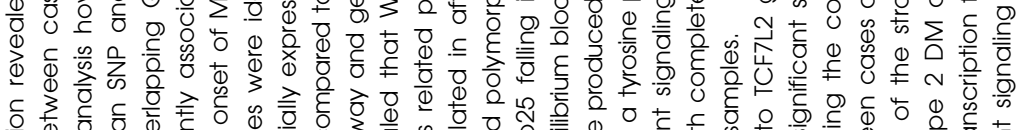

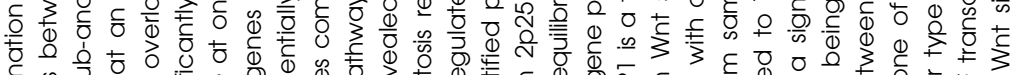

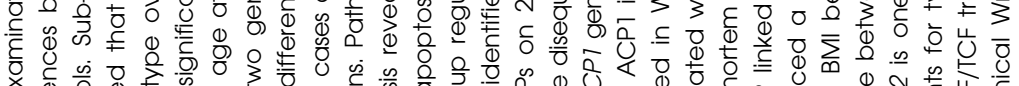

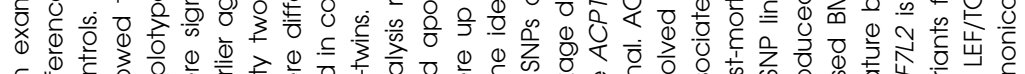

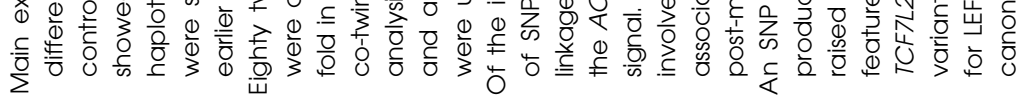

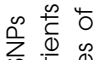

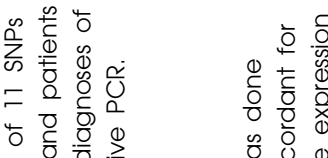

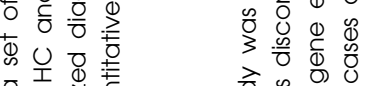

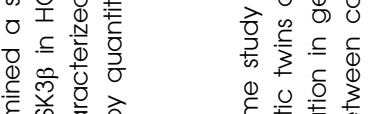

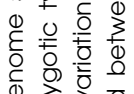

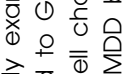

궁하일

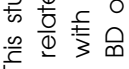

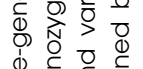

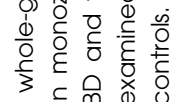

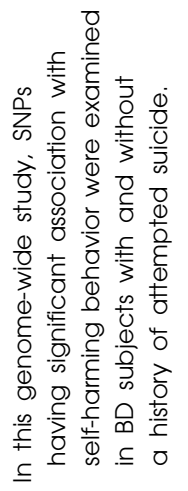

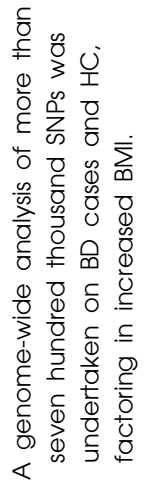

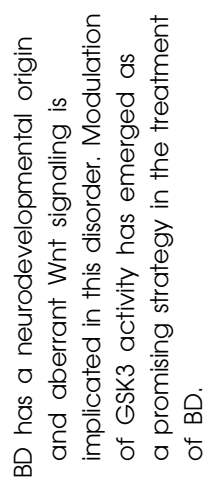

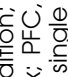

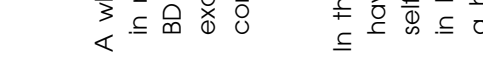
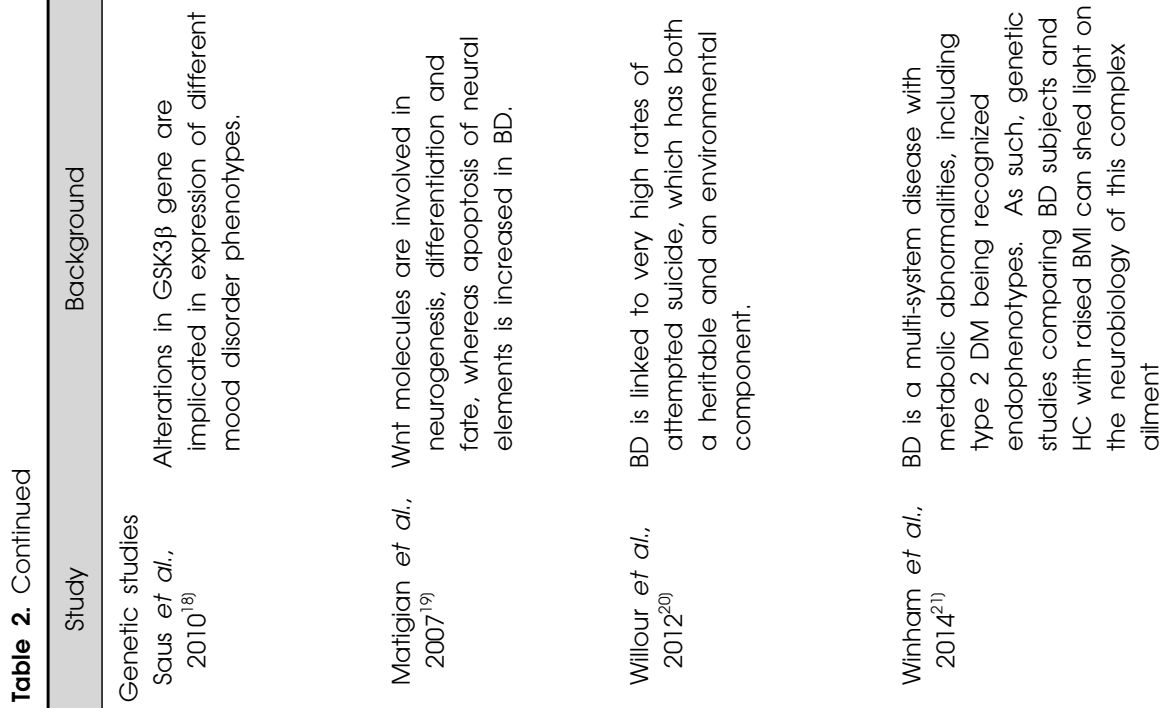

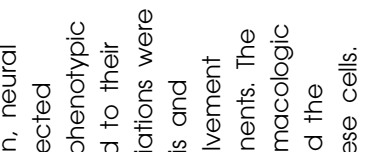

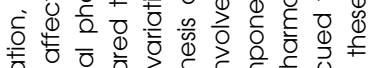

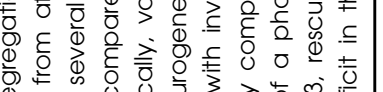

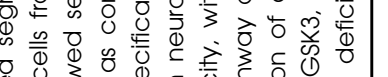

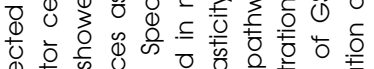

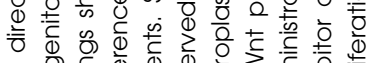

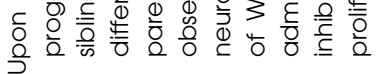

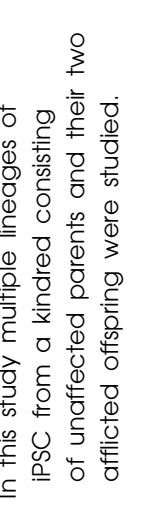

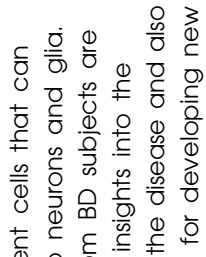

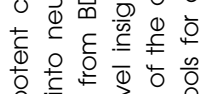

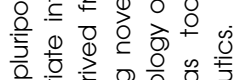

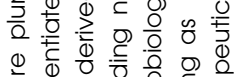

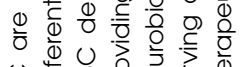

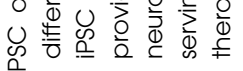

要

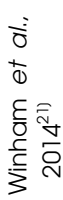

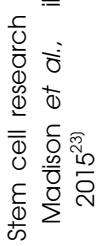

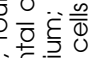

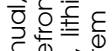

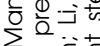

$\overline{0} \overline{0} . \overline{0}$

它

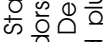

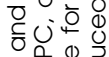

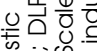

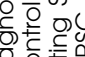

00

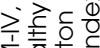

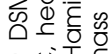

호오둘

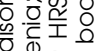

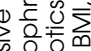

运:

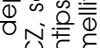

을

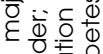

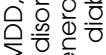

$\sum_{i=0}$ 흥

ه

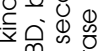

क्षे

高

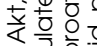

is $\overline{0} \frac{0}{0} \overline{0}$

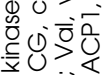

0
0
0

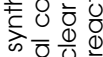

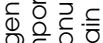

0

के द्ध

굽응

प)

is 흥

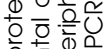

응으.

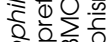

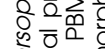

○े 齐

舟

흥 응

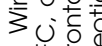

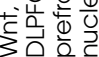


the lateral ventricles in the forebrain. NPC generated in the SGZ migrate into the granular layer of the dentate gyrus where they mature and integrate into the existing neuronal circuitry, whereas those originating from SVZ migrate anteriorly through the rostral migratory stream and differentiate into interneurons in the olfactory bulb. Neurogenesis is decreased in mood disorders, but is promoted by mood stabilizing drugs, foremost example being lithium. Inactivation of GSK3 modulates the differentiation and integration of NPC in the adult brain and transgenic mice (Ser21/9Ala) have deficits in proliferation and maturation of neural precursors. This evidence points to the fact that mood stabilizers, at least in part work through enhancing neurogenesis via inhibition of GSK3. ${ }^{26)}$ Several transcription factors, for example c-Myc, c-Jun, Klf5 and snail which are linked to cell fate are directly phosphorylated by GSK3 leading to their ubiquitination and proteosomal degradation. While this is the case in mature, differentiated cells under basal conditions, the activity of GSK3 is much lower in stem/precursor cells due to the action of growth factors and Wnt molecules. Therefore, in BD over activity of GSK3 may undermine neurogenesis, and psychotropic agents by enhancing this process promote mood stabilization. ${ }^{27)}$ Further, detailed characterization of this mode of action of mood stabilizing medications can result in targeted therapeutics of BD.

\section{GSK3 and Inflammation}

Among the downstream actions of GSK3, the most crucial effect is the control of more than 50 transcription factors that are responsible for many different cellular functions. It has recently being shown that this kinase effects the production of pro-inflammatory cytokines, as well as promoting the differentiation of pro-inflammatory subtypes of T-helper cells. Inflammatory mediators are being increasingly incriminated in mood disorders; in this regard the current evidence suggests that a constant low-grade inflammatory state persists that causes neuroprogression and also underlies the many comorbidities associated with these conditions. ${ }^{28)}$ GSK3 is thought to increase the phosphorylation of nuclear factor kappa B and signal transducer and activator of transcription 3 , which are the main regulators of the production of pro-inflammatory cytokines, at the same time inhibiting cAMP response element-binding protein and activator protein 1 which effect expression of anti-inflammatory cytokine, interleukin (IL)-10. mTOR pathway has been shown to decrease inflammatory cytokine production by inactivat- ing GSK3, while inhibitors of this enzyme rescue mice from an otherwise lethal dose of lipopolysacchride. ${ }^{29)}$ To sum up, in BD increased activity of GSK3 may underpin enhanced expression of key inflammatory mediators like IL-6 and tumor necrosis factor $\alpha$ during affective exacerbations, and inhibition of this enzyme by mood stabilizers leads to the resolution of the inflammatory state.

\section{GSK3 and Neuroprotection}

There is accumulating evidence of increase in the process of programmed cell death or apoptosis in BD. Post-mortem studies indicate that there is a $27 \%$ deficit of gabaergic interneurons in the brain; additionally, proapoptotic genes are over-expressed while anti-apoptotic proteins like BDNF and B-cell lymphoma-2 have a reduced expression. ${ }^{30)}$ GSK3 appears to have a dual role in apoptosis as this kinase promotes cell death caused by the mitochondrial intrinsic apoptotic pathway, but inhibits the death-receptor mediated extrinsic apoptotic signaling pathway. By inactivating the transcription mediator heat shock factor-1, GSK3 reduces the expression of chaperone proteins, a key cellular protective mechanism against neuronal insults, and inhibitors of this enzyme bolster cellular responses to stress by upregulating the expression of chaperone proteins. ${ }^{31)}$

\section{Role of GSK3 in Synaptic Plasticity}

A growing body of research advocates that affective, cognitive and behavioral abnormalities in mood disorders arise from defective information processing in critical synapses and circuits. ${ }^{32)}$ There are long lasting changes in brain function resulting from variation in signal strength at synapses, epitomized by the processes of long-term potentiation and depression (LTP, LTD). GSK3 is a potent regulator of transmission across synapses, and by thus influencing synaptic plasticity it plays a crucial role in neuropsychiatric disorders. ${ }^{33)}$ At the presynapse, high GSK3 activity reduces glutamate release, antagonizing LTP and at this site its prominent target is dynamin-1, a large GTPase that controls vesicle endocytosis. Phosphorylation of dynamin-1 by GSK3 is required for efficient retrieval and recycling of synaptic vesicles and it is likely that cycles of phosphorylation/dephosphorylation of this and other trafficking proteins are required for proper neurotransmission. ${ }^{34)}$ Evidence also points out that voltage gated channels are regulated by GSK3, albeit in an indirect manner. For instance, the cytoskeleton-associated protein collapsin 2 is a GSK3 substrate that increases cell surface expression of $\mathrm{CaV} 2.2$, promoting neurotransmitter re- 
lease from the presynapse. Further, GSK3 mediated phosphorylation of collapsin 2 reduces binding to tubulin heterodimers, decreases their polymerization into microtubules and diminishes neurite outgrowth. As such, in mood disorders and schizophrenia, elevated GSK3 activity would be expected to decrease dendritic arborization and possibly synapse formation, which may contribute to the pathogenesis of these disorders. ${ }^{35)}$ At the postsynapse, GSK3 has been found to regulate the cell surface expression of glutamate and 5HT receptors, as well as calcium channel proteins. The antimanic agents lithium and valproate, but not the antidepressant imipramine, decrease synaptic GluA1 levels, suggesting that regulation of glutamatergic signaling at the postsynapse is clinically significant. Several GABA-A cell surface receptors, such as CACNA1b, CACNA1c and HCN4 are genetically associated with mood disorders and schizophrenia, and it will be interesting to determine whether GSK3 and its clinical inhibitors modulate the expression and activity of these as well. $^{36)}$

\section{GSK3 and the Circadian Clock}

Among the core disturbances in mood disorders, circadian rhythm abnormality can be considered as the most significant as it is present not only during the acute phase but persists in the inter-episode period as well. In BD, disruption of the daily rhythm is most often associated with affective exacerbations, while aberrations in the sleepwake schedule are integral to the diagnosis of both mania and depression. The circadian clock is inherent to every cell in the body, but the hypothalamic supraschiasmatic nucleus ( $\mathrm{SCN}$ ) is the master pacemaker. The precise SCN rhythm is controlled by cyclic transcription, translation and degradation of a group of transcription factors and signaling proteins. ${ }^{37)}$ Briefly, the clock is kicked off by two transcription factors, CLOCK and BMAL1 which heterodimerize and bind to Enhancer-box sequences in promoter regions of clock-controlled genes, namely Period (Per) and Cryptochromes (Cryl and Cry2). These are transcriptional repressors and translocate to the nucleus, where they bind to the CLOCK/BMAL1 and inhibit transcription of CLOCK/E-box controlled genes. Next in sequence, PER and CRY are phosphorylated and degraded, thus reactivating the transcription of E-box genes and resetting the circadian clock. Intriguingly, GSK3 is reported to phosphorylate nearly all of these clock-associated proteins. Phosphorylation of CLOCK, BMAL1 and CRY2 regulate their degradation or stability, whereas phosphorylation of PER facilitates its translocation to the nucleus. ${ }^{38)}$
As of now, none of these substances have been validated as physiologic substrates of GSK3 and this matter awaits verification. Nonetheless, the modulation of clock proteins links with cyclic regulation of GSK3 activity via its inhibitory N-terminal serine phosphorylation through PI3K-Akt pathway. GSK3 knockin mice (Ser21/9Ala) show lengthening of their circadian cycle, while selective GSK3 inhibitors or RNA interference shorten the period. In contrast, lithium treatment lengthens the circadian period in mammals which may be because this psychotropic medication's effect on the internal clock is independent of GSK3. Nonetheless, it is highly likely that GSK3 mediated phosphorylation of some circadian proteins contributes to the development of mood disorders and schizophrenia. $^{39)}$

Figure 3 provides an outline of the upstream effectors and downstream regulators of GSK3 activity implicated in the pathophysiology of mood disorders.

\section{Psychotropic Drugs Act through Wht and GSK3 Signaling Pathways}

$\mathrm{BD}$ is a difficult to treat condition and the majority of patients need more than one medication for symptomatic relief. Acute episodes, whether manic, depressive or mixed can present management challenges so that a combination of mood stabilizers and atypical antipsychotics is frequently employed to induce remission. Antidepressant monotherapy is regarded as mood destabilizing and present consensus dictates that these medications should be used adjunctively to mood stabilizers, which are considered the primary pharmacotherapuetic strategy for all phases of the disorder. Figure 4 gives a schematic depiction of the current understanding of the pharmacological treatment of BD. Accumulating data over the past two decades indicates that Wnt/GSK3 signaling pathway is fundamentally involved in the mechanism of action of these medications and this section of the manuscript provides a brief overview of the existing literature in this regard.

\section{Lithium and GSK3}

Lithium both directly inhibits GSK3 by competing for a magnesium binding site and indirectly by N-terminal serine phosphorylation of the kinase. This action has been demonstrated in human PBMC, as well as in the mouse brain. The inhibitory serine phosphorylation may be mediated by disruption of a protein complex consisting of $\beta$-arrestin 2/Akt/PP2 or an indirect inactivation of protein phosphatase $1 .^{40)}$ Lithium attenuates locomotor hyper- 


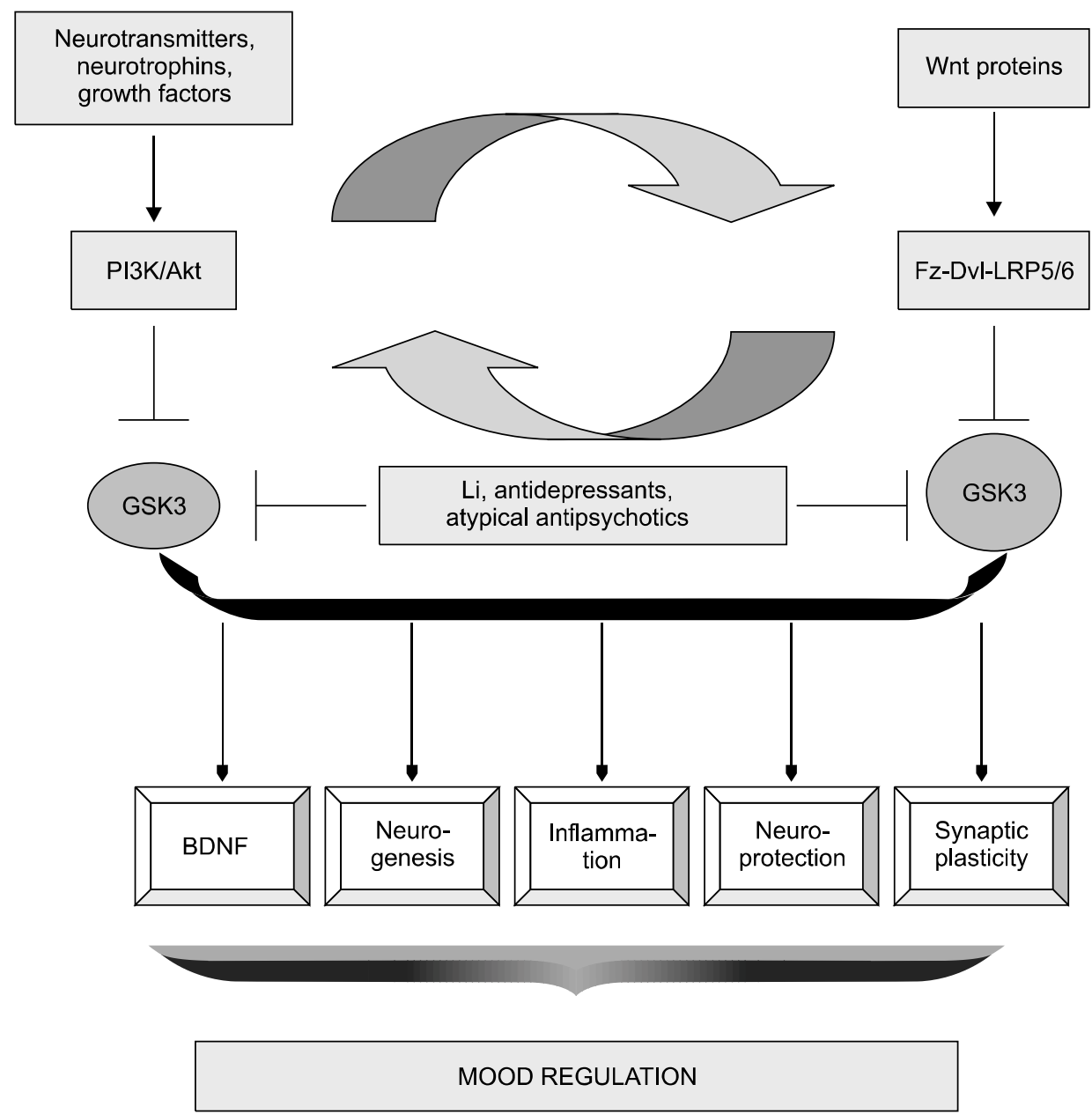

Fig. 3. Up- and down-stream modulators of glycogen synthase kinase (GSK) $3 \beta$ that regulate mood. GSK3 is hyperactivated in major mood disorders, downstream of monoaminergic signaling, DISCl, neuregulin and brain derived neurotrophic factor (BDNF). These diverse mediators converge through the PI3K/AKt cascade and lead to the inhibition of GSK3. In addition, wingless Drosophila proteins (Wnt) molecules acting via their transmembrane receptors and cytosolic effectors also inactivate GSK3. There is cross-talk between these two pathways by means of mammalian target of rapamycin (MTOR) and heat inducible factor (HIF) $1 \alpha$; mTOR is activated by Akt which promotes HIF- $1 \alpha$ translation, increasing expression of Wnt target genes. The downstream modulators of GSK3 $\beta$ have crucial mood regulating effects through augmented mechanisms which include increased neurotrophic support, dendritic growth and arborization and enhanced synaptic plasticity. This process of neuroprotection is promoted by psychotropic drugs by virtue of their inhibitory action on GSK3 activity.

Akt, protein kinase B; DISC1, disrupted in schizophrenia 1; Dvl, dishevelled; Fz, frizzled; Li, lithium; LRP, low density lipoprotein receptor related protein; PI3K, phosphoinositide 3-kinase.

activity in response to novelty in Clock-mutant mice which are advanced as a rodent model of bipolar mania. ${ }^{41)}$ Further, lithium treatment blocks stress-induced depressive-like behaviors in pre-pubertal and adult rats subjected to chronic mild stress, with changes in the expression of GSK $3 \beta$ and its target genes in the hippocampus. ${ }^{42)}$ The current evidence suggests that GSK3 and $\beta$-catenin are nodal points in the development of mood disorders and this pathway is primarily involved in the therapeutic effects of lithium in affective illnesses.

\section{Anticonvulsant Mood Stabilizers}

Anticonvulsants are increasingly being employed in the treatment of $\mathrm{BD}$, as monotherapy or in conjunction with atypical antipsychotics during acute affective exacerbations, as well as in the maintenance phase of the disorder. Valproate which is a simple branched-chain fatty acid has established efficacy in manic, mixed and depressive episodes, and although with regards to GSK3 inhibition there is conflicting evidence, it is a well-studied histone deacetylase (HDAC) inhibitor. HDAC inhibition increases Akt phosphorylation, with resulting inactivation of GSK3 and this mechanism enhances the mood stabilizing effect of 


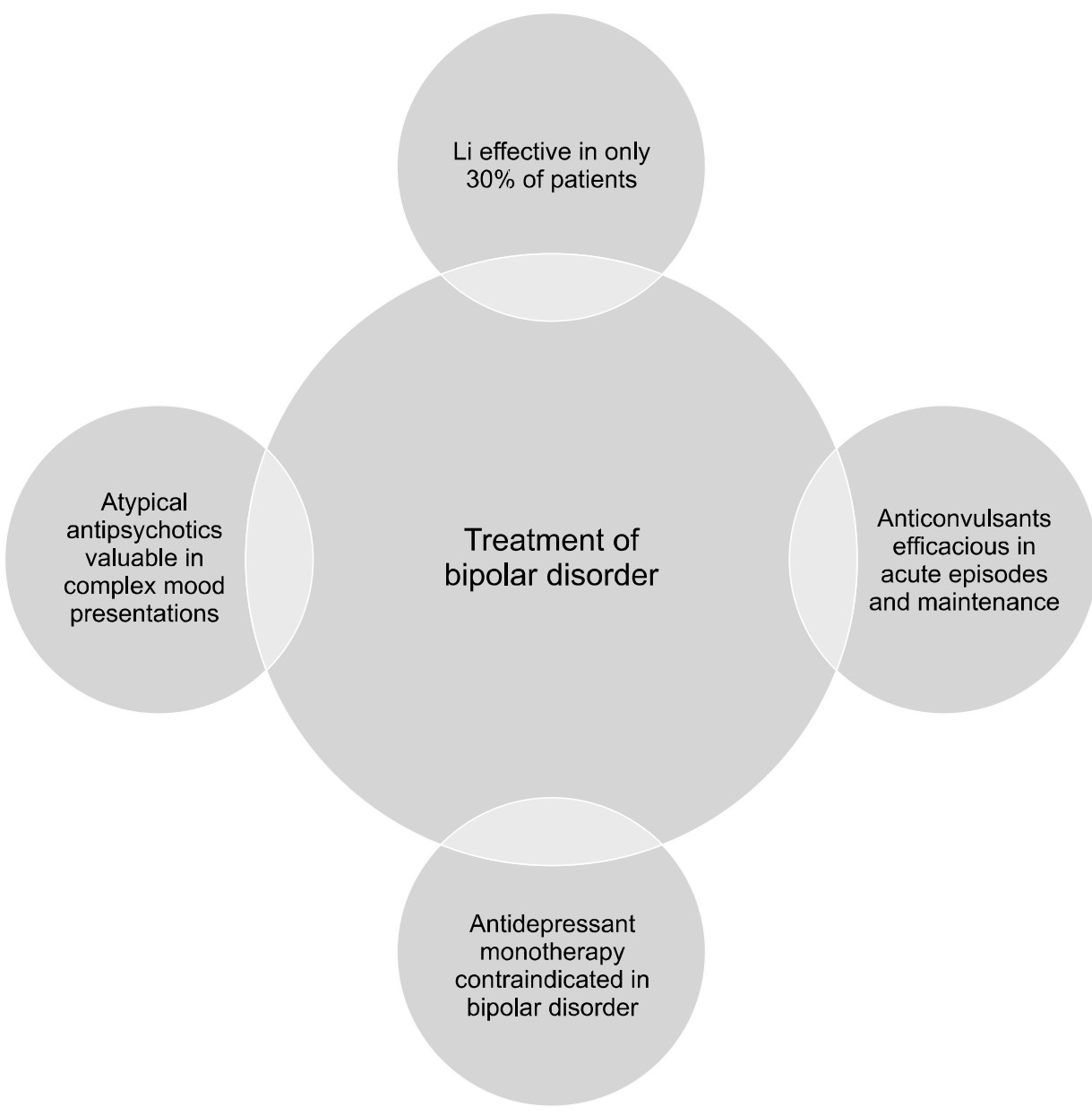

Fig. 4. Pharmacotherapy of bipolar disorder. Bipolar disorder is a difficult to treat condition, as affective symptoms may persist during the inter-episode period. Less than one third of patients show responsiveness to the classical mood stabilizer, lithium (Li). New generation anticonvulsants are being employed with increasing frequency with favorable efficacy and safety profile. Atypical antipsychotics control core affective symptoms and are also useful in comorbid anxiety and substance use disorders. Antidepressants employed as monotherapy are liable to induce mixed states, subthreshold symptoms and rapid cycling.

lithium. ${ }^{43)}$ In addition, chronic valproate treatment has been found to induce changes in the level of microRNAs (miRNAs) in the rat hippocampus, and the targets of some of these miRNAs are proteins involved in the canonical Wnt pathway. ${ }^{44)}$ With respect to other antiepileptics frequently used in BD like carbamazepine and lamotrigine published data does not support GSK3 inhibition, however these and other new generation anticonvulsants may have mood regulating effect by virtue of their actions on targets up- or down-stream of this kinase.

\section{Atypical Antipsychotics}

SGAs are of paramount significance in the treatment of mood disorders in general and $\mathrm{BD}$, in particular. Most SGAs are regarded as first line agents in the therapy of manic and mixed episodes and some are indicated in bipolar depression. There is emerging evidence of their efficacy in the maintenance treatment of BD either as monotherapy or as adjuncts to mood stabilizers. A cardinal difference between SGAs and conventional antipsychotics is the formers' ability to block 5HT2 receptors in addition to antagonizing dopamine D2 receptors (D2R). There is convincing proof that SGAs modulate GSK $3 \alpha / \beta$ activity by inhibitory ser $21 / 9$ phosphorylation. ${ }^{45)}$ A further advancement in the treatment of major mental disorders is exemplified by so called 'third generation antipsychotics' represented by aripiprazole, brexpiprazole, and cariprazine. These novel drugs have been shown to possess biased signaling characteristics or functional selectivity at G-protein coupled receptors (GPCR), namely the D2R. Downstream signaling at the GPCR can be mediated by protein kinase A (PKA) or Akt, the later being kept in a dephosphorylated state in a protein complex which also includes the scaffolding protein, $\beta$-arrestin 2 and GSK3 $\beta$. Biased ligands like aripiprazole can differentially activate the D2R, either through PKA or Akt/ $\beta$-arrestin $2 /$ GSK3 $\beta$ cascade, ultimately causing the transcription of Wnt related genes via $\beta$-catenin. ${ }^{46)}$ This mechanism is of key importance in the treatment of intractable mental illnesses including MDD, BD and schizophrenia, and several drug 
molecules in development are exploiting biased signaling at the $\mathrm{D} 2 \mathrm{R}$ with this rationale.

\section{Antidepressants and GSK3}

Antidepressants are sometimes used to alleviate depressive states in BD in conjunction with mood stabilizers, this strategy being employed to prevent the induction of mixed phenomena and rapid cycling. There is a slowly growing body of evidence which indicates that monoamine modulating antidepressants indeed target Wnt pathway components in the brain and while GSK3 inhibition by these psychotropic agents occurs acutely, the onset of therapeutic effect takes weeks. This alludes to the fact that changes in neuroplasticity that are dependent on gene transcription occur slowly, resulting in antidepressant action in weeks to months. Continued intakes of psychotropic medications results in incremental benefits in all realms of functioning, as new neuronal connections are formed that improve psychopathological and psychosocial illness domains. In brief, psychotropic drugs enhance neuronal survival and growth and Wnt and GSK3 related cellular cascades apparently play a crucial role in this process. $^{47)}$

Table 3 sums up the effects of psychotropic drugs on GSK3 and Wnt cellular cascades.

\section{Therapeutic Potential of GSK3 Modulation in Bipolar Disorder}

There is now ample evidence that GSK3 over activity is present in BD and this perturbation can induce both manic and depressive type behaviors. Dysregulated activity of the kinase can act through diverse paths in causing the development and progression of this and other major psychi- atric disorders. ${ }^{48)}$ For instance in patients experiencing repeated mood episodes, there is exacerbation of the proinflammatory state, increased oxidative stress and decline in neurotrophic support leading to increased apoptosis of neural elements. In this regard the neuroprotective action of lithium is well documented, so that the challenge for future is the development of promising new drugs based on GSK3 inhibition which are disease specific, have an improved safety and tolerability profile and produce an ameliorative effect on illness pathogenic mechanisms. ${ }^{49)}$ Based on this rationale novel compounds have been discovered which can potentially lead to new avenues in the treatment of $\mathrm{BD}$ and provide cures for the illness and its many comorbidities. ${ }^{50)}$ The test for the coming era is to continue endeavors in this regard so that new therapeutic agents can be brought into clinical practice for a recalcitrant disease.

\section{CONCLUSION}

$\mathrm{BD}$ is presently regarded as a systemic ailment with numerous adverse sequelae in the biopsychosocial realm. The etiopathogenic mechanisms underpinning this disease are hitherto unknown; however, the diathesis exerts a pervasive negative effect on all bodily systems. Presently available treatments only have a palliative effect and there is an urgent need for medications that have actual curative value. The first mood stabilizing medicine lithium was discovered serendipitously; however, the finding that it was an inhibitor of the enzyme glycogen synthase kinase 3 opened new areas of research. Since then an ever increasing body of work has elucidated the roles of GSK3 and the associated Wnt pathways in mood disorders and

Table 3. Psychotropic drug effects on Wnt associated molecules

\begin{tabular}{|c|c|c|c|}
\hline Medication & GSK3 $\beta$ ser9 phosphorylation & $\begin{array}{c}\text { Protein kinase B or Akt } \\
\text { phosphorylation }\end{array}$ & $\beta$-catenin levels \\
\hline Lithium & $\uparrow$ & Conflicting evidence & $\uparrow$ \\
\hline Valproate & $\uparrow$ & $\uparrow$ & $\uparrow$ \\
\hline Carbamazepine & Conflicting evidence & No data & Conflicting evidence \\
\hline Lamotrigine & $\uparrow$ (unpublished observations) & No data & No data \\
\hline Clozapine & $\uparrow$ & $\uparrow$ & $\uparrow$ \\
\hline Risperidone & $\uparrow$ & Conflicting data & $\uparrow$ \\
\hline Olanzapine & $\uparrow$ & No data & No data \\
\hline Quetiapine & $\uparrow$ & No data & No data \\
\hline Ziprasidone & $\uparrow$ & No data & No data \\
\hline Fluoxetine & $\uparrow$ & No data & $\uparrow$ \\
\hline Fluvoxamine & No data & $\uparrow$ (via sigma-1 receptor) & $\uparrow$ \\
\hline Sertraline & No data & $\downarrow$ & No data \\
\hline Venlafaxine & No data & No data & $\uparrow$ \\
\hline
\end{tabular}

Wnt, Wingless Dorsophila proteins; GSK3, glycogen synthase kinase 3; Akt, protein kinase B. 
several other neuropsychiatric ailments. The pivotal role of these cellular cascades in psychiatric disorders is well established and increasing knowledge has led to a better understanding of the neurobiology of major mental diseases. New therapies based on this knowhow have the potential to bring important advances in the treatment of mental illnesses in general and BD in particular. As such, the test for the future is to elucidate the mechanisms that lead to the development of principal psychiatric disorders and discover disease specific therapeutic agents that can alleviate the suffering of innumerable people afflicted by these diseases.

\section{REFERENCES}

1. Muneer A. Staging models in bipolar disorder: a systematic review of the literature. Clin Psychopharmacol Neurosci 2016;14:117-130.

2. Muneer A. The neurobiology of bipolar disorder: an integrated approach. Chonnam Med J 2016;52:18-37.

3. Cole AR. Glycogen synthase kinase 3 substrates in mood disorders and schizophrenia. FEBS J 2013;280:5213-5227.

4. Uribe E, Wix R. Neuronal migration, apoptosis and bipolar disorder. Rev Psiquiatr Salud Ment 2012;5:127-133.

5. Valvezan AJ, Klein PS. GSK-3 and Wnt signaling in neurogenesis and bipolar disorder. Front Mol Neurosci 2012;5:1

6. Golpich M, Amini E, Hemmati F, Ibrahim NM, Rahmani $\mathrm{B}$, Mohamed Z, et al. Glycogen synthase kinase-3 beta (GSK-3 $\beta$ ) signaling: implications for Parkinson's disease. Pharmacol Res 2015;97:16-26.

7. Beurel E, Grieco SF, Jope RS. Glycogen synthase kinase-3 (GSK3): regulation, actions, and diseases. Pharmacol Ther 2015; 148:114-131.

8. Ka M, Condorelli G, Woodgett JR, Kim WY. mTOR regulates brain morphogenesis by mediating GSK3 signaling. Development 2014;141:4076-4086.

9. Durak O, de Anda FC, Singh KK, Leussis MP, Petryshen TL, Sklar P, et al. Ankyrin-G regulates neurogenesis and Wnt signaling by altering the subcellular localization of $\beta$-catenin. Mol Psychiatry 2015;20:388-397.

10. Jope RS. Glycogen synthase kinase-3 in the etiology and treatment of mood disorders. Front Mol Neurosci 2011;4:16.

11. Rajkowska G. Cell pathology in bipolar disorder. Bipolar Disord 2002;4:105-116.

12. Karege F, Perroud N, Burkhardt S, Schwald M, Ballmann E, La Harpe R, et al. Alteration in kinase activity but not in protein levels of protein kinase $B$ and glycogen synthase kinase-3beta in ventral prefrontal cortex of depressed suicide victims. Biol Psychiatry 2007;61:240-245.

13. Pandey GN, Rizavi HS, Tripathi M, Ren X. Region-specific dysregulation of glycogen synthase kinase- $3 \beta$ and $\beta$-catenin in the postmortem brains of subjects with bipolar disorder and schizophrenia. Bipolar Disord 2015;17:160-171.

14. Ren X, Rizavi HS, Khan MA, Dwivedi Y, Pandey GN. Altered Wnt signalling in the teenage suicide brain: focus on glycogen synthase kinase- $3 \beta$ and $\beta$-catenin. Int $J$ Neuropsychopharmacol 2013;16:945-955.

15. Polter A, Beurel E, Yang S, Garner R, Song L, Miller CA, et al. Deficiency in the inhibitory serine-phosphorylation of glycogen synthase kinase-3 increases sensitivity to mood disturbances. Neuropsychopharmacology 2010;35:1761-1774.

16. Li X, Liu M, Cai Z, Wang G, Li X. Regulation of glycogen synthase kinase-3 during bipolar mania treatment. Bipolar Disord 2010;12:741-752.

17. de Sousa RT, Zanetti MV, Talib LL, Serpa MH, Chaim TM, Carvalho AF, et al. Lithium increases platelet serine-9 phosphorylated GSK-3 $\beta$ levels in drug-free bipolar disorder during depressive episodes. J Psychiatr Res 2015;62:78-83.

18. Saus E, Soria V, Escaramís G, Crespo JM, Valero J, Gutiérrez-Zotes A, et al. A haplotype of glycogen synthase kinase $3 \beta$ is associated with early onset of unipolar major depression. Genes Brain Behav 2010;9:799-807.

19. Matigian N, Windus L, Smith H, Filippich C, Pantelis C, McGrath J, et al. Expression profiling in monozygotic twins discordant for bipolar disorder reveals dysregulation of the WNT signalling pathway. Mol Psychiatry 2007;12:815-825.

20. Willour VL, Seifuddin F, Mahon PB, Jancic D, Pirooznia $\mathrm{M}$, Steele $\mathrm{J}$, et al. A genome-wide association study of attempted suicide. Mol Psychiatry 2012;17:433-444.

21. Winham SJ, Cuellar-Barboza AB, Oliveros A, McElroy SL, Crow S, Colby $\mathrm{C}$, et al. Genome-wide association study of bipolar disorder accounting for effect of body mass index identifies a new risk allele in TCF7L2. Mol Psychiatry 2014; 19:1010-1016.

22. O'Shea KS, McInnis MG. Neurodevelopmental origins of bipolar disorder: iPSC models. Mol Cell Neurosci 2016; 73:63-83.

23. Madison JM, Zhou F, Nigam A, Hussain A, Barker DD, Nehme R, et al. Characterization of bipolar disorder patient- specific induced pluripotent stem cells from a family reveals neurodevelopmental and $m R N A$ expression abnormalities. Mol Psychiatry 2015;20:703-717.

24. Gould TD. Targeting glycogen synthase kinase-3 as an approach to develop novel mood-stabilising medications. Expert Opin Ther Targets 2006;10:377-392.

25. Machado-Vieira R, Manji HK, Zarate CA Jr. The role of lithium in the treatment of bipolar disorder: convergent evidence for neurotrophic effects as a unifying hypothesis. Bipolar Disord 2009;11 Suppl 2:92-109.

26. Ferensztajn-Rochowiak E, Rybakowski JK. The effect of lithium on hematopoietic, mesenchymal and neural stem cells. Pharmacol Rep 2016;68:224-230.

27. Hussaini SM, Choi CI, Cho CH, Kim HJ, Jun H, Jang MH. Wnt signaling in neuropsychiatric disorders: ties with adult hippocampal neurogenesis and behavior. Neurosci Biobehav Rev 2014;47:369-383.

28. Muneer A. Bipolar disorder: role of inflammation and the development of disease biomarkers. Psychiatry Investig 2016;13:18-33.

29. Ajmone-Cat MA, D'Urso MC, di Blasio G, Brignone MS, De Simone R, Minghetti L. Glycogen synthase kinase 3 is part of the molecular machinery regulating the adaptive response to LPS stimulation in microglial cells. Brain Behav Immun 2016;55:225-235.

30. Viswanath B, Jose SP, Squassina A, Thirthalli J, Purushottam M, Mukherjee O, et al. Cellular models to study bipolar disorder: a systematic review. J Affect Disord 2015;184: 36-50.

31. Li X, Bijur GN, Jope RS. Glycogen synthase kinase-3 beta, mood stabilizers, and neuroprotection. Bipolar Disord 2002;4:137-144.

32. Du J, Machado-Vieira R, Khairova R. Synaptic plasticity in the pathophysiology and treatment of bipolar disorder. Curr Top Behav Neurosci 2011;5:167-185.

33. Ochs SM, Dorostkar MM, Aramuni G, Schön C, Filser S, 
Pöschl J, et al. Loss of neuronal GSK3 $\beta$ reduces dendritic spine stability and attenuates excitatory synaptic transmission via $\beta$-catenin. Mol Psychiatry 2015;20:482-489.

34. Smillie KJ, Cousin MA. Akt/PKB controls the activitydependent bulk endocytosis of synaptic vesicles. Traffic 2012;13:1004-1011.

35. Beurel E. HDAC6 regulates LPS-tolerance in astrocytes. PLoS One 2011;6:e25804.

36. Kalkman HO. Potential opposite roles of the extracellular signal-regulated kinase (ERK) pathway in autism spectrum and bipolar disorders. Neurosci Biobehav Rev 2012;36: 2206-2213.

37. Besing RC, Paul JR, Hablitz LM, Rogers CO, Johnson RL, Young ME, et al. Circadian rhythmicity of active GSK3 isoforms modulates molecular clock gene rhythms in the suprachiasmatic nucleus. J Biol Rhythms 2015;30:155-160.

38. Paul JR, Johnson RL, Jope RS, Gamble KL. Disruption of circadian rhythmicity and suprachiasmatic action potential frequency in a mouse model with constitutive activation of glycogen synthase kinase 3. Neuroscience 2012;226:1-9.

39. McCarthy MJ, Wei H, Marnoy Z, Darvish RM, McPhie DL, Cohen BM, et al. Genetic and clinical factors predict lithium's effects on PER2 gene expression rhythms in cells from bipolar disorder patients. Transl Psychiatry 2013;3: e318.

40. O'Brien WT, Huang J, Buccafusca R, Garskof J, Valvezan AJ, Berry GT, et al. Glycogen synthase kinase-3 is essential for $\beta$-arrestin-2 complex formation and lithium-sensitive behaviors in mice. J Clin Invest 2011;121:3756-3762.

41. Kozikowski AP, Gunosewoyo H, Guo S, Gaisina IN, Walter $\mathrm{RL}$, Ketcherside $\mathrm{A}$, et al. Identification of a glycogen synthase kinase- $3 \beta$ inhibitor that attenuates hyperactivity in CLOCK mutant mice. ChemMedChem 2011;6:1593-1602.

42. O'Leary OF, O'Connor RM, Cryan JF. Lithium-induced effects on adult hippocampal neurogenesis are topographically segregated along the dorso-ventral axis of stressed mice. Neuropharmacology 2012;62:247-255.

43. Kao CY, Hsu YC, Liu JW, Lee DC, Chung YF, Chiu IM. The mood stabilizer valproate activates human FGF1 gene promoter through inhibiting HDAC and GSK-3 activities. $J$ Neurochem 2013;126:4-18.

44. Zhou R, Yuan P, Wang Y, Hunsberger JG, Elkahloun A, Wei $\mathrm{Y}$, et al. Evidence for selective microRNAs and their effectors as common long-term targets for the actions of mood stabilizers. Neuropsychopharmacology 2009;34:13951405.

45. Kalinichev M, Dawson LA. Evidence for antimanic efficacy of glycogen synthase kinase-3 (GSK3) inhibitors in a strain-specific model of acute mania. Int $J$ Neuropsychopharmacol 2011;14:1051-1067.

46. de Bartolomeis A, Tomasetti C, Iasevoli F. Update on the mechanism of action of aripiprazole: translational insights into antipsychotic strategies beyond dopamine receptor antagonism. CNS Drugs 2015;29:773-799.

47. Pilar-Cúellar F, Vidal R, Díaz A, Castro E, dos Anjos S, Vargas V, et al. Signaling pathways involved in antidepressant-induced cell proliferation and synaptic plasticity. Curr Pharm Des 2014;20:3776-3794.

48. Del'Guidice T, Latapy C, Rampino A, Khlghatyan J, Lemasson M, Gelao B, et al. FXR1P is a GSK3 $\beta$ substrate regulating mood and emotion processing. Proc Natl Acad Sci U S A 2015;112:E4610-E4619.

49. Meffre D, Grenier J, Bernard S, Courtin F, Dudev T, Shackleford $\mathrm{G}$, et al. Wnt and lithium: a common destiny in the therapy of nervous system pathologies? Cell Mol Life Sci 2014;71:1123-1148.

50. Furlotti G, Alisi MA, Cazzolla N, Dragone P, Durando L, Magarò $\mathrm{G}$, et al. Hit optimization of 5-substituted-N(piperidin-4-ylmethyl)-1H-indazole-3-carboxamides: potent glycogen synthase kinase-3 (GSK-3) inhibitors with in vivo activity in model of mood disorders. $J$ Med Chem 2015;58: 8920-8937. 